\title{
Fast approximate 2D inversion of airborne TEM data: Born approximation and empirical approach
}

\author{
Julien Guillemoteau*, Pascal Sailhac* and Mickael Behaegel ${ }^{\dagger}$
}

\begin{abstract}
Airborne transient electromagnetic surveying provides data sections with a sufficient coverage to perform $2 \mathrm{D}$ imaging of electrical conductivity within the ground. Full 2D inversion using numerical modeling with finite differences or finite elements is still a time-consuming method to process the large amount of data acquired during an airborne survey. 2D structures increase the complexity of eddy current patterns within the ground. Consequently, fast approximate imaging using 2D sensitivities of equivalent homogeneous media is not sufficient and causes strong artefacts in the resulting model. To overcome this problem, one prefers to use 1D inversion or $3 \mathrm{D}$ inversion using local sensitivity to process this kind of data. However, we consider a fast 2D inversion to be reachable. By estimating numerically the $2 \mathrm{D}$ sensitivity caused by $2 \mathrm{D}$ perturbations and showing that it differs considerably from the ones derived from homogeneous media, we propose an empirical model for in-loop configuration which describes the numerical 2D sensitivity. By applying this method to synthetic data, we show that it eliminates $2 \mathrm{D}$ artefacts which are often encountered when using approximate inverse methods based on the theory of equivalent homogeneous halfspace. An application to real in-loop data illustrates this improvement for imaging a dipping layer of conductive graphite deposits in Canada. This method is relatively fast. It could provide a better understanding of the ground during the survey and would allow geophysicists to better manage the whole campaign.
\end{abstract}

* Institut de Physique du Globe de Strasbourg UMR 7516; Universite de Strasbourg/EOST, CNRS; 5 Rue Rene Descartes, 67000 Strasbourg, France

$\dagger$ Areva NC, Geoscience Direction, Mining Business Group, La Defense, Paris, France

\section{INTRODUCTION}

The Airborne ElectroMagnetic method (AEM) operating in time domain is a practical tool to map near-surface geological features over large areas (several tens of kilometres). The principal applications are mining exploration (Smith et al., 2010; Wolfgram and Golden, 2001), subsurface hydrology (Danielsen et al., 2003; Baldridge et al., 2007; Auken et al., 2009; Siemon et al., 2009; Kirkegaard et al., 2011) and on-shore hydrocarbon exploration (Huang and Rudd, 2008; Pfaffhuber et al., 2009). AEM data are often interpreted using apparent conductivity transforms (Wolfgram and Karlik, 1995; Macnae et al., 1998), equivalent plate inversion (Keating and Crossley, 1990; Tartaras et al., 2000; Zhdanov et al., 2002), diffusion velocity analysis (Eaton and Hohmann, 1987), Zohdy's method (Zohdy, 1989; Sattel, 2005) or layered earth inversion (Huang and Palacky, 1991; Chen and Raiche, 1998; Farquharson et al., 1999; Christensen, 2002; Viezzoli et al., 2008; Vallee and Smith, 2009; Guillemoteau et al., 2011). All of these methods are cost effective and allow geophysicists to rapidly process the large amount of data acquired during airborne surveys. Also, a fast 3D EM inversion algorithm has recently been presented for frequency domain data (Cox et al., 2010). However, most of the surveys have been acquired along flight lines that have considerable distance between them. The information contained within such data sets does not allow the 3D model equivalences to be resolved. Consequently, 3D inversion still requires a prior model provided by $1 \mathrm{D}$ interpretation or else strong regularization of the model parameters. In the presence of high lateral conductivity contrasts, 1D interpretation may lead to strong artefacts in the resulting subsurface image (Ley-Cooper et al., 2010).

An intermediate solution is to interpret the data using a 2D theory. Full 2D inversion using finite elements has been presented by Wilson et al. (2006) but this method is not fast enough to process large amounts of data. The fast approximate inverse mapping (AIM) approach (Old- 
enburg and Ellis, 1991), which uses the Born approximation applied to an equivalent homogeneous medium, has been tested by Wolfgram et al. (2003) for the 2D inversion of airborne TEM data. The method does not however reproduce the true conductivity when inverting only the vertical component of the magnetic field.

In this paper, we first examine the theory of approximate inverse mapping applied to the $2 \mathrm{D}$ subsurface imaging where the sensitivity function is derived from the BiotSavart law. Secondly, we determine numerically the 2D sensitivity function due to $2 \mathrm{D}$ conductivity perturbations for the in-loop configuration and show that it differs considerably from the sensitivity derived using the AIM method. As a consequence, we suggest a new approximate theory which better describes how the ground is illuminated during in-loop TEM acquisition. Finally, we compare the new method with 1D and 2D AIM inversion as applied to two kinds of data sets: synthetic data with a pure step waveform and actual helicopter borne data with a realistic waveform.

\section{THEORY AND METHODS}

The transient electromagnetic method involves exciting the ground with a time varying loop source and measuring the magnetic field resulting from induced currents in the ground (Palacky, 1981; Nabighian and Macnae, 1988). In order to understand how the ground is illuminated during an airborne TEM measurement, one has to study the sensitivity function or the Frechet kernel. Let us recall the basic principles of imaging in order to define the $2 \mathrm{D}$ TEM inverse problem.

\section{Forward modeling using the Born Approx- imation}

The inversion of TEM data consists in determining the subsurface distribution of electrical conductivity from measurements of the decaying vertical magnetic field after the source is turned off. The 3D forward problem can be written as follows (Gòmez-Treviño, 1987a,b):

$$
h_{z}\left(\mathbf{r}_{R}, t\right)=\iiint_{V} g(\mathbf{r}, t, \sigma) \sigma(\mathbf{r}) d V
$$

where $h_{z}\left(\mathbf{r}_{R}, t\right)$ is the decaying magnetic field measured at receiver position $\mathbf{r}_{R}=\left\{x_{R}, y_{R}, z_{R}\right\}$ and $g$ is the sensitivity function or Frechet kernel. The sensitivity function depends on the acquisition geometry and on the distribution of conductivity in the ground. Since the distribution of conductivity is the sought-after parameter, the inverse problem is nonlinear.

For a heterogeneous media, the function $g$ has to be computed using a numerical method such as finite differences or finite elements. However these methods are too time consuming for practical inversion. One can reduce the computational time by using approximate inverse mapping as introduced by Oldenburg and Ellis (1991). This method consists in applying the perturbation theory

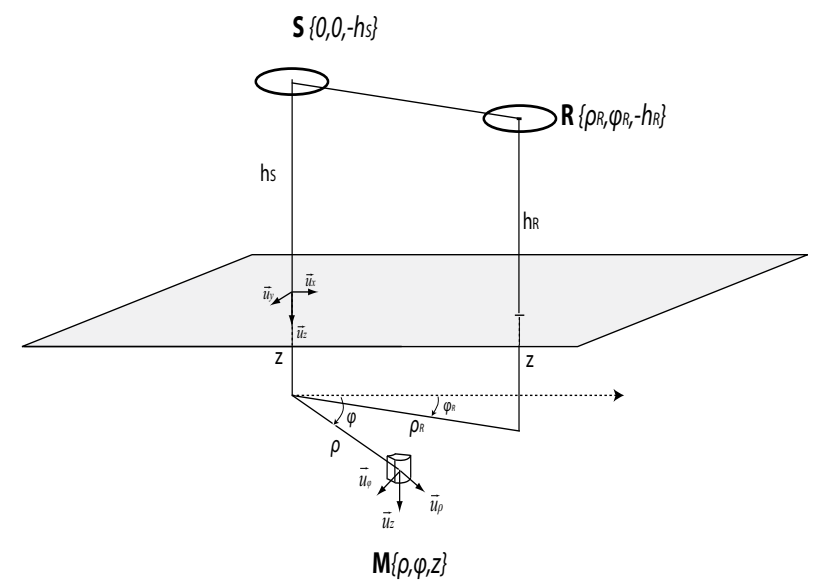

Figure 1: Schematic description of EM measurement with source $S$ and receiver $R$ in the air.

based on an equivalent homogeneous half-space. In such case, the transient magnetic response can be written as follows:

$h_{z}\left(\mathbf{r}_{R}, t\right)=h_{z}^{0}\left(\mathbf{r}_{R}, t\right)+\iiint_{V} g\left(\mathbf{r}, t, \sigma_{0}\right)\left[\sigma(\mathbf{r})-\sigma_{0}(\mathbf{r})\right] d v$

where $\sigma_{0}(\mathbf{r})=\sigma_{a}$ is the conductivity of the equivalent homogeneous medium with transient magnetic response $h_{z}^{0}$. This approximation works well only if the actual subsurface does not differ strongly from the homogeneous model. The problem can be simplified in this case using the sensitivity function associated with the initial model:

$$
h_{z}\left(\mathbf{r}_{R}, t\right)=\iiint_{V} g\left(\mathbf{r}, t, \sigma_{0}\right) \sigma(\mathbf{r}) d v .
$$

Approximate inverse mapping has been applied to the 1D inversion of TEM data by Christensen (1995). Data can be associated to the conductivity of the equivalent homogeneous half space which would have produce the same response. By considering this apparent conductivity $\sigma_{a}$ as data which controls the scaling of the sensitivity, the $3 \mathrm{D}$ equivalent problem may be written as follows:

$$
\sigma_{a}\left(\mathbf{r}_{R}, t\right)=\iiint_{V} f\left(\mathbf{r}, t, \sigma_{a}\right) \sigma(\mathbf{r}) d v
$$

where

$$
f\left(\mathbf{r}, t, \sigma_{a}\right)=\frac{\partial \sigma_{a}\left(\mathbf{r}_{R}, t\right)}{\partial h_{z}\left(\mathbf{r}_{R}, t\right)} g\left(\mathbf{r}, t, \sigma_{a}\right) .
$$

The function $f$ can be viewed as a spatial averaging of the conductivity describing how the ground is illuminated for a given source-receiver configuration. It depends on both the eddy currents caused by the source and the properties of the receiver. The magnetic response at the receiver position can be estimated using the Biot-Savart law (see Figure 1):

$$
\vec{h}\left(r_{R}, t\right)=\frac{1}{4 \pi} \iiint_{V} \frac{\vec{j}\left(\mathbf{r}, t ; \mathbf{r}_{S}\right) \wedge \overrightarrow{M R}\left(\mathbf{r}, \mathbf{r}_{R}\right)}{\left\|\overrightarrow{M R}\left(\mathbf{r}, \mathbf{r}_{R}\right)\right\|^{3}} d V
$$




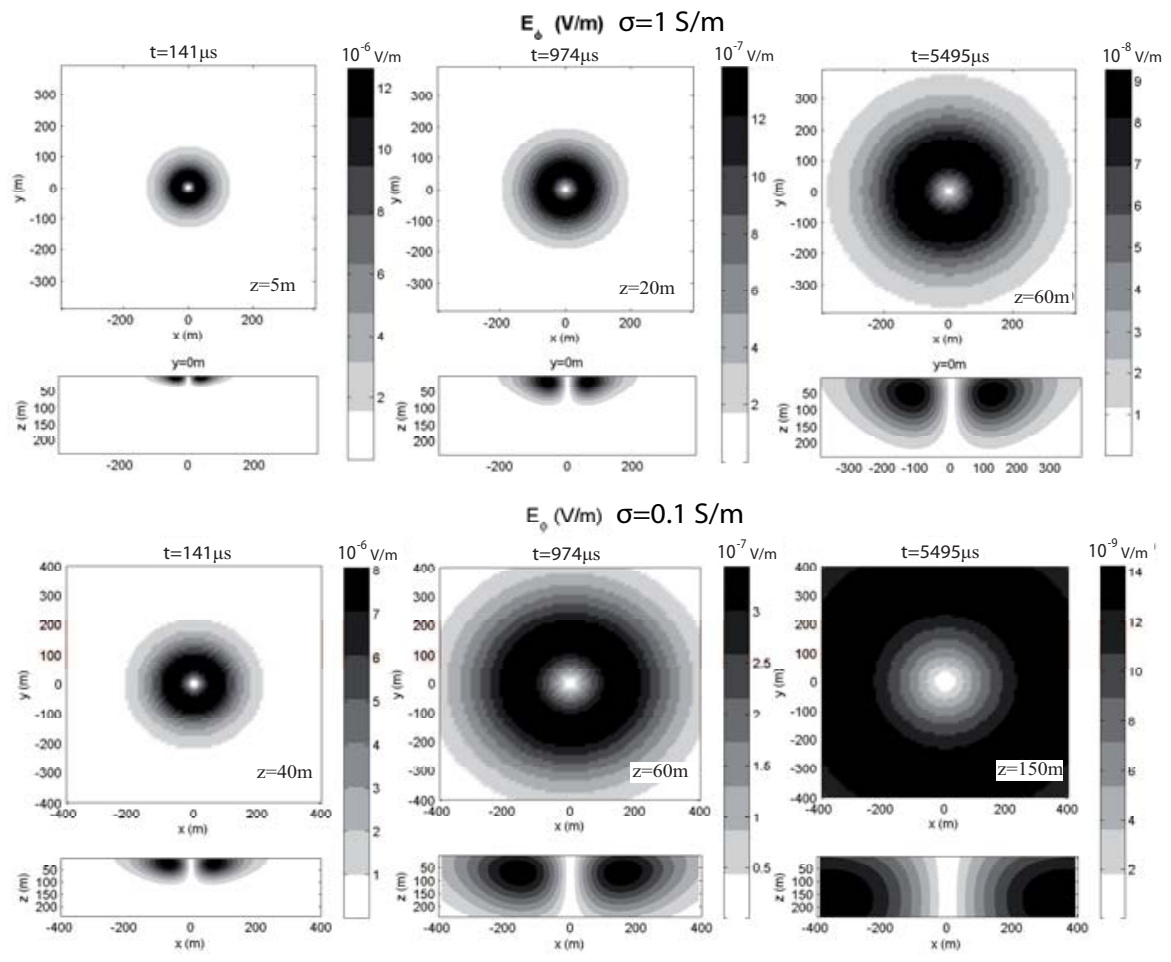

Figure 2: Spatial distribution of $e_{\phi}$ in the ground for three channels after the 1 A current has been turned off. The loop source has a radius $a=13 \mathrm{~m}$ and is located at $h_{S}=45 \mathrm{~m}$. Upper part: $\sigma=1 \mathrm{~S} / \mathrm{m}$, lower part: $\sigma=0.1 \mathrm{~S} / \mathrm{m}$.

where $\mathbf{r}_{S}$ is the position of the source and $\overrightarrow{M R}$ is the vector between the receiver and the volume element of secondary source with the current density $\vec{j}$. For a homogeneous halfspace, eddy currents flow in horizontal loops. Therefore, in cylindrical coordinates,

$\vec{j}(\mathbf{r}, t)=\|\vec{j}(\mathbf{r}, t)\| \cdot\left(\begin{array}{c}-\sin \phi \\ \cos \phi \\ 0\end{array}\right) \overrightarrow{M R}=\left(\begin{array}{c}\rho_{R} \cos \phi_{R}-\rho \cos \phi \\ \rho_{R} \sin \phi_{R}-\rho \sin \phi \\ z_{R}-z\end{array}\right)$

where $\|\vec{j}\|$ is the norm of the current density depending on both conductivity and the transient electrical field $\vec{e}_{\phi}(\mathbf{r}, t)$ induced within the ground:

$$
\|\vec{j}(\mathbf{r}, t)\|=\sigma(\mathbf{r})\left\|\vec{e}_{\phi}(\mathbf{r}, t)\right\| .
$$

In a cylindrical system oriented downward, the transient electrical field in the homogeneous half space due to a horizontal loop source in the air (TE mode) points in the azimuthal direction $\vec{u}_{\phi}$ and is given in the frequency domain by (see Appendix A):

$$
E_{\phi}^{s t e p}(\omega, \rho, z)=\frac{\mu_{0} I a}{2} \int_{0}^{\infty} \frac{t_{T E}}{u_{1}} e^{-u_{0} h_{S}} e^{-u_{1} z} \lambda J_{1}(\lambda a) J_{1}(\lambda \rho) d \lambda,
$$

where $\mu_{0}=4 \pi 10^{-7} \mathrm{H} / \mathrm{m}$ is the magnetic permeability of free space, $a$ is the radius of the loop source, $I$ is the intensity of the electrical current in the loop source, $u_{0}$ and $u_{1}$ are the wave number for the air and the half space respectively, $h_{S}$ is the height of the source, $\rho$ is the horizontal distance between the source and the receiver, and $t_{T E}$ is the transmission coefficient for the TE mode given by:

$$
t_{T E}=\frac{2 u_{1}}{u_{0}+u_{1}} \text {. }
$$

In Figure 2, we show the spatial distribution of the electrical field in time domain computed by setting $s=i \omega$ and by performing an inverse Laplace transform of equation 9. As expected, the results show that ground induced currents diffuse downward like a smoke ring (Nabighian, 1979; Reid and Macnae, 1998) which becomes smoother with increasing time. The diffusion velocity of the electrical field decreases in more conductive media. Using the equations 6 and 7 , the sensitivity function for the three components $(n=x, y, z)$ of the magnetic field in the air is estimated by:

$$
h_{n}\left(\mathbf{r}_{R}, t\right)=\iiint_{V}\|\vec{j}(\mathbf{r}, t)\| R_{n}\left(\mathbf{r}, \mathbf{r}_{R}\right) d x d y d z,
$$

where $R_{n}$ is given for the three components of the Cartesian coordinates system:

$$
\begin{aligned}
& R_{x}(x, y, z)=\frac{1}{4 \pi \sqrt{x^{2}+y^{2}}} \frac{\left(z_{R}-z\right) x}{\left(\left(x_{R}-x\right)^{2}+\left(y_{R}-y\right)^{2}+\left(z_{R}-z\right)^{2}\right)^{\frac{3}{2}}}, \\
& R_{y}(x, y, z)=\frac{1}{4 \pi \sqrt{x^{2}+y^{2}}} \frac{\left(z_{R}-z\right) y}{\left(\left(x_{R}-x\right)^{2}+\left(y_{R}-y\right)^{2}+\left(z_{R}-z\right)^{2}\right)^{\frac{3}{2}}}, \quad(13) \\
& R_{z}(x, y, z)=\frac{1}{4 \pi \sqrt{x^{2}+y^{2}}} \frac{x^{2}+y^{2}-x x_{R}-y y_{R}}{\left(\left(x_{R}-x\right)^{2}+\left(y_{R}-y\right)^{2}+\left(z_{R}-z\right)^{2}\right)^{\frac{3}{2}}} .
\end{aligned}
$$



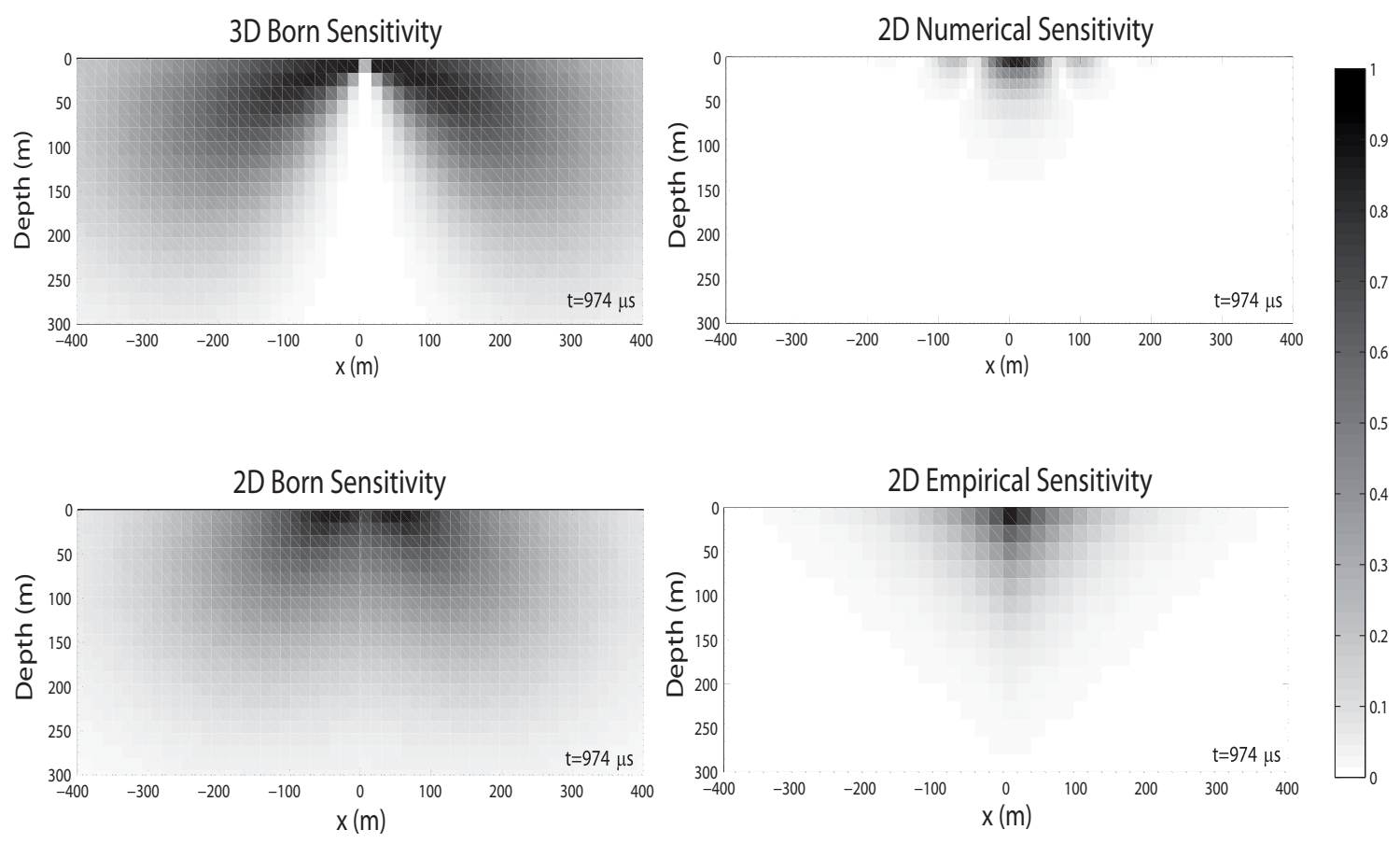

Figure 3: Left column: sensitivity functions derived using the Biot-Savart law and the Born approximation. Right column: sensitivity functions derived from numerical modeling (top) and empirical approach (bottom). The loop source of radius $a=13 \mathrm{~m}$ is located at $h_{S}=45 \mathrm{~m}$ over a half-space with the conductivity $\sigma=10^{-2} \mathrm{~S} / \mathrm{m}$.

By comparing (3), (11) and (8), we can see that the sensitivity function $g$ is the product between the electrical field in a homogeneous media and a geometric function $R_{n}$ given by (12)-(14), which describes the field of view of the receiver:

$$
g_{n}\left(\mathbf{r}, t ; \sigma_{a}, \mathbf{r}_{R}, \mathbf{r}_{S}\right)=\left\|\vec{e}_{\phi}\left(\mathbf{r}, t ; \sigma_{a}, \mathbf{r}_{S}\right)\right\| \cdot R_{n}\left(\mathbf{r} ; \mathbf{r}_{R}\right) .
$$

If one knows the 3D spatial distribution of eddy currents, one can compute the 3D sensitivity function for a homogeneous medium by using the equation 15 . The 3D inversion process is time consuming because it requires many model parameters and data acquisition with 3D coverage. Therefore, 2D inversion appears as an intermediate method that would produce imaging results relatively quickly with fewer parameters. As in Wolfgram et al. (2003), our 2D sensitivities are computed by integration of the $3 \mathrm{D}$ sensitivity over the invariant y-direction (strike). In Figure 3, we show the spatial distribution of the $3 \mathrm{D}$ and $2 \mathrm{D}$ sensitivity of the vertical magnetic field for a zero offset configuration. The main consequence of using a $2 \mathrm{D}$ sensitivity instead of a $3 \mathrm{D}$ sensitivity is that conductive structures not directly beneath the flight line can get mapped as a deeper body in the resulting $2 \mathrm{D} \mathrm{sec-}$ tion. It is also important to note that the Biot-Savart law is valid in free space. A more accurate approach is to compute the secondary magnetic field for a half-space using electrical dipole sources to simulate the induced currents. The free space sensitivity has similar shape but its spatial extent is overestimated compared to the half-space sensitivity. We correct this for difference by multiplying the apparent conductivity by 4 in order to get the same spatial extent as for half-space sensitivity.

\section{Forward modeling using an empirical ap- proach}

Wolfgram et al. (2003) applied an adaptive Born approximation to 2D inversion of airborne TEM data. They show that it is difficult to constrain a 2D model by using only the vertical component of the magnetic field $H_{z}$. We suggest that the approximate inverse mapping using a homogeneous half-space oversimplifies the pattern of eddy currents in a 2D structure. Indeed, as 2D objects have strike length much longer than the spatial footprint, they may generate even stronger perturbations than those caused by a $3 \mathrm{D}$ body. Using the program ArjunAir developed at CSIRO (Raiche, 2008), we computed the 2D sensitivity function by considering local 2D perturbations $\Delta \sigma$ successively located at all positions within the ground. The computation of this numerical kernel is carried out in the following three steps:

1 First, magnetic responses are computed numerically with ArjunAir for each of perturbations.

2 Second, the magnetic responses are converted to apparent conductivities by using a table look-up method.

3 Finally, the sensitivity function is computed by using the following equation:

$$
f(x, z, t)=\frac{\Delta \sigma_{a}(x, z, t)}{\Delta \sigma},
$$


where $\Delta \sigma_{a}(x, z, t)$ is the difference between the apparent conductivity of the unperturbed medium and a medium with a perturbation $\Delta \sigma$ at the position $(x, z)$. In Figure 3 (top right), the right numerical $2 \mathrm{D}$ kernel is shown for a perturbation embedded within a homogeneous medium of conductivity $\sigma=10^{-2} \mathrm{~S} / \mathrm{m}$. It differs considerably from the kernel obtained by the AIM theory derived for an equivalent homogeneous half-space (see left part of Figure 3). There is one important difference that affects the
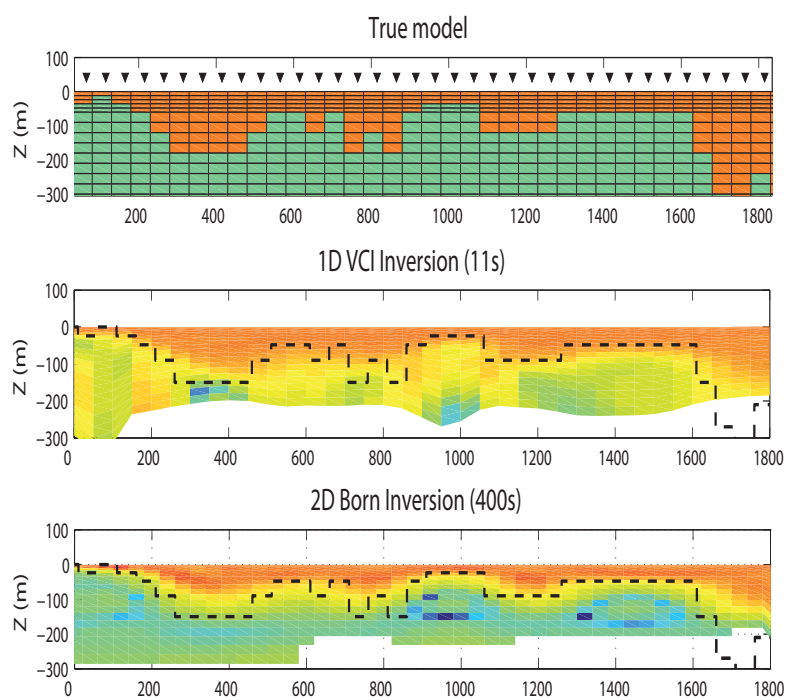

2D Empirical Inversion (80s)

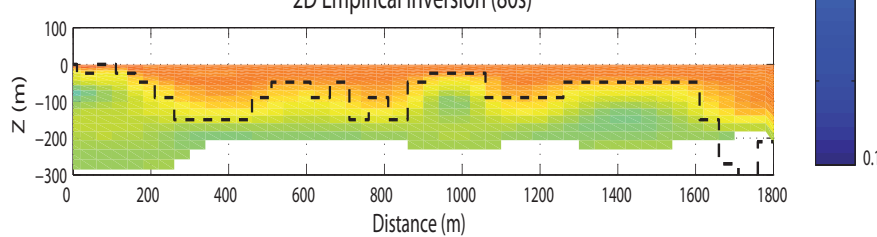

Figure 4: Inversion of the VTEM synthetic data generated by a weakly $2 D$ model with a shallow conductive layer. From top to bottom: true model and position of soundings (triangles); concatenated result of $1 D$ inversions using vertical constraints (VCI); result of the 2D Born inversion using the Biot-Savart sensitivity; result of the $2 D$ empirical inversion. The computational times, associated to a Matlab code running with one processor of $2.39 \mathrm{GHz}$ and 3.45 Go of RAM, is given in second for each method.

inversion: the standard AIM sensitivities are characterized by two symmetric lobes at the centre of central loop system, while the numerical one shows a dominant single lobe centred just beneath the system and two smaller sides lobes. This method cannot be implemented for fast $2 \mathrm{D}$ inversion, however, as the computations are too timeconsuming. Following Christensen (2002) for 1D TEM imaging, we suggest an empirical model to approximate the real 2D kernel. Consider the illuminated domain defined as $\Omega_{i}=\left\{\left[-d_{x}, d_{x}\right],\left[0, d_{z}\right]\right\}$ where $d_{z}$ and $d_{x}$ are the distances of penetration in the vertical and horizontal directions, respectively. We find that the sensitivity function of the vertical magnetic field can be approximated by a simple mathematical formula:

$$
\begin{aligned}
& f(x, z, t)=\frac{1}{\Gamma} \begin{cases}\exp -\left(\frac{4|x|}{d_{x}}+\frac{6 z}{d_{z}}\right) & ,(x, z) \in \Omega_{i} . \\
0 & , \text { elsewhere. }\end{cases} \\
& d_{z}=\sqrt{\frac{c_{z} t}{\mu_{0} \sigma_{a}(t)}} \text { and } d_{x}=\sqrt{\frac{c_{x} t}{\mu_{0} \sigma_{a}(t)}}+h_{R},
\end{aligned}
$$

where $c_{z}$ is the scaling factor for the vertical direction defined by Christensen (2002), $c_{x}$ is the scaling factor for nooo the horizontal direction, $h_{R}$ is the receiver height and $\Gamma$ is a normalizing factor detailed below. As the kernel for the apparent conductivity has to follow the condition:

$$
\iint_{\Omega} f\left(x, z, \sigma_{a}, h_{R}\right) d x d z=1
$$

the new empirical kernel is normalized by:

$$
\Gamma=\iint_{\Omega_{i}} \exp -\left(\frac{4|x|}{d_{x}}+\frac{6 z}{d_{z}}\right) d x d z .
$$

This approximate kernel is valid for the in-loop configuration only. It is shown at the bottom right of Figure 3 for the time channel $t=974 \mu \mathrm{s}$ and the $45 \mathrm{~m}$ transmitter height over a half-space of conductivity $\sigma=10^{-2} \mathrm{~S} / \mathrm{m}$. Using this approach, we assume that a cell with anomalous conductivity is unaffected by the presence of another. In other words, this method does not include the mutual inductance.

\section{Inverse Problem}

The data for the inversion scheme consists of the apparent conductivity versus time while the model is the spatial distribution of conductivity in a vertical plane beneath the flight line. The apparent conductivities are computed prior to the inversion by looking in tables which contain the responses of the system for a large range of homogeneous earth models and transmitter heights. The response of the system is obtained by convolving the time derivative of the source current with the step response. In the case of an in-loop system and sufficiently late time channels, the $B_{z}$ response is monotonic with regards to conductivity, therefore there is a unique correspondence between the apparent conductivity and the response. The 2D linear inverse problem is formulated as follows:

$$
\sigma_{a}\left(\mathbf{r}_{R}, t\right)=\iint_{\Omega} f\left(\mathbf{r}, t, \sigma_{a}\right) \sigma(\mathbf{r}) d r
$$

where $f\left(\mathbf{r}, t, \sigma_{a}\right)$ is the $2 \mathrm{D}$ sensitivity function given by equation 17. If we consider $N_{s}$ soundings acquired at different positions along the flight line, with each sounding recorded at $N_{t}$ time windows, the data set is composed of $N_{d}=N_{s} \times N_{t}$ data. By discretizing the 2D space into 

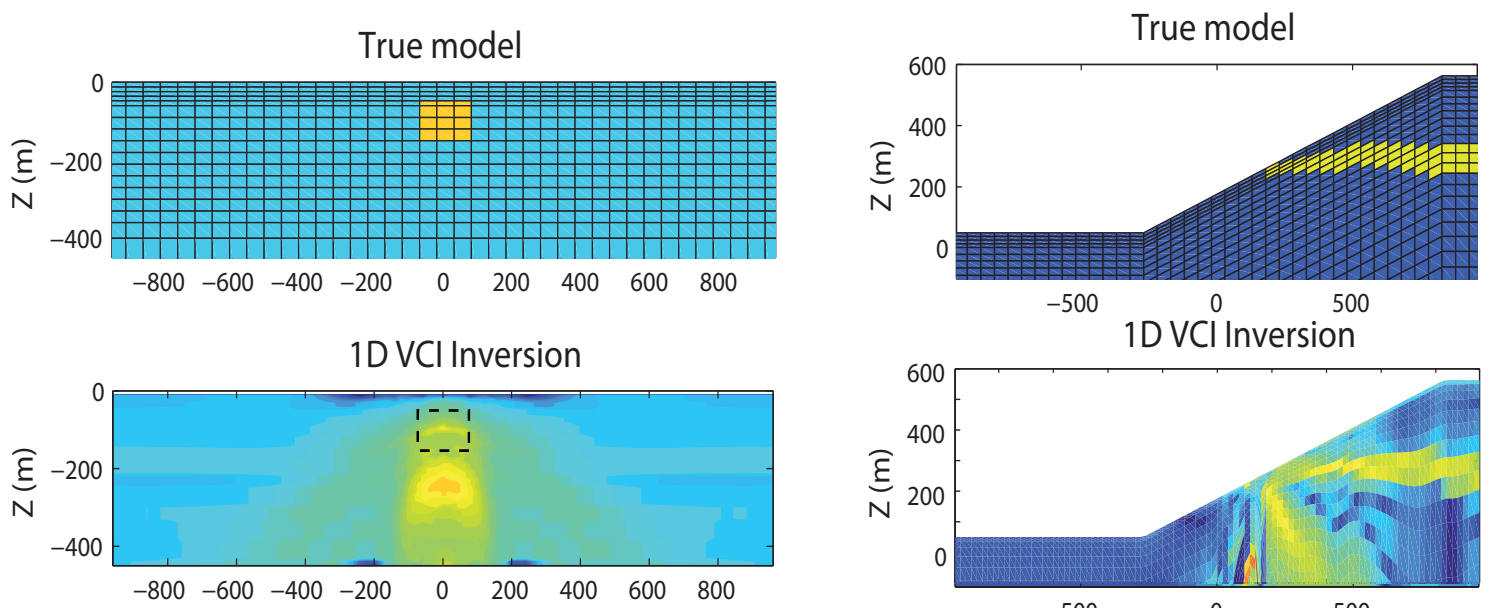

$\sigma(\mathrm{mS} / \mathrm{m})$
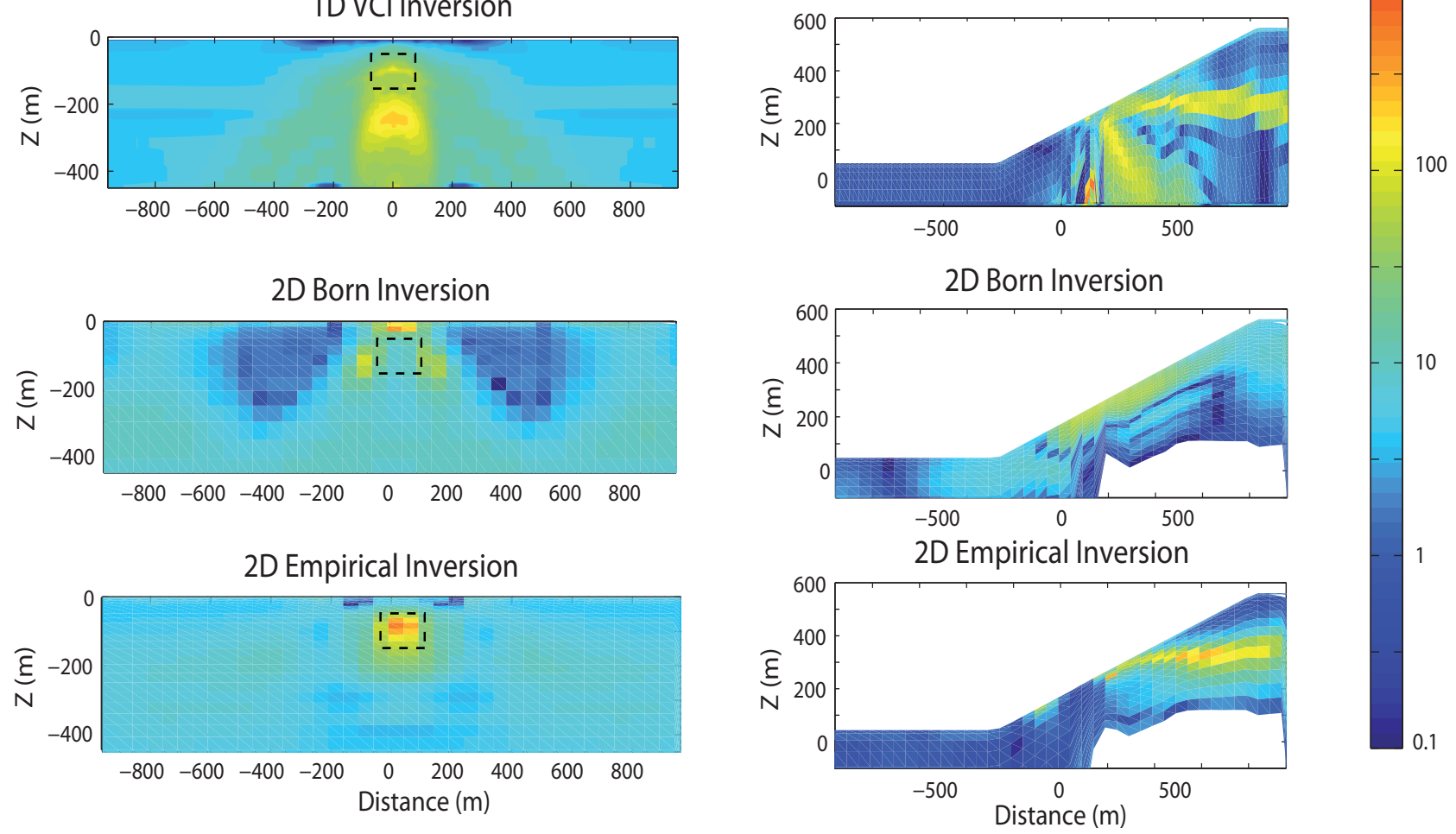

Figure 5: Inversion of VTEM synthetic data. Left column: small prism $(100 \mathrm{~m} \times 100 \mathrm{~m})$ with a conductivity of 200 $\mathrm{mS} / \mathrm{m}$ embedded in a half-space with a conductivity of $5 \mathrm{mS} / \mathrm{m}$. Right column: horizontal layer with a conductivity of $100 \mathrm{mS} / \mathrm{m}$ embedded in resistive medium with a conductivity of $1 \mathrm{mS} / \mathrm{m}$ and simple topography. From top to bottom: cross section of the true model, after 1D inversion, after $2 D$ Born inversion, and after $2 D$ empirical inversion. 
$N_{m}$ blocks, we can write the inverse problem in a matrix form as follows:

$$
\sigma_{a, i}=\sum_{j} \mathbf{F}_{i, j} \sigma_{j} \quad i=1, N_{d} \quad j=1, N_{m},
$$

where $\mathbf{F}_{i, j}$ contains the integral of $f\left(\mathbf{r}, t, \sigma_{a}\right)$ over each the $j_{t h}$ model block for the $i_{t h}$ data point. Our inversion scheme is separated into two steps. First, we solve equation set 22 using a Tikhonov regularization strategy:

$$
\left[\begin{array}{c}
\mathbf{F} \\
\mathbf{S}_{x} \\
\mathbf{S}_{z}
\end{array}\right] \sigma=\left[\begin{array}{c}
\sigma_{\mathbf{a}} \\
\mathbf{0} \\
\mathbf{0}
\end{array}\right]
$$

The matrix $\mathbf{F}$ is related to the data misfit; $\mathbf{S}_{x}$ and $\mathbf{S}_{z}$ are smoothness matrices which constrain, respectively, the horizontal and vertical second derivatives of the recovered model. We solve this problem using the Conjugate Gradient Least Square method (CGLS) (Aster et al., 2005; Hansen, 2010). The initial model is formed by equating the conductivity with the apparent conductivity at the centroid depth of each measurement. If the conductivity of the host rock is known, we use it instead. Secondly, we perform the additional recursive filter presented in Guillemoteau et al. (2011) which re-weights the inverse problem in the logarithmic space:

$$
\sigma=\sigma^{\mathbf{0}} e^{\left[\mathbf{F}^{* T} \mathbf{W}_{d} \mathbf{F}^{*}+\lambda_{p}^{2} \mathbf{I}\right]^{-1} \mathbf{F}^{*} \mathbf{W}_{d} \log \left(\sigma_{\mathbf{a}} / \sigma_{\mathbf{a}}^{\mathbf{o}}\right)},
$$

where $\sigma^{0}$ is the initial conductivity which is, at the first iteration, the solution of the equation set 23 with large smoothness. $\lambda_{P}$ is a Lagrangian parameter which controls the difference with the starting model $\sigma^{0}$ at each iteration, $\sigma_{a}$ is the measured apparent conductivity, $\sigma_{\mathbf{a}}^{\mathbf{0}}=\mathbf{F} \sigma^{\mathbf{0}}$ is the predicted apparent conductivity, $\mathbf{W}_{d}$ is a diagonal error matrix with elements proportional to $h_{z}(t)$. As $h_{z}(t)$ is a decaying curve with regard to the time, this advantages the short time channel fitting. $\mathbf{F}^{*}$ is the logarithmic kernel which is expressed as:

$$
F_{i j}^{*}=\frac{\sigma_{j}^{0}}{\sigma_{a, i}} F_{i j} .
$$

The computation of the logarithmic kernel $\mathbf{F}^{*}$ is very fast at each step as we already know the standard kernel $\mathbf{F}$. The parameter $\lambda_{P}$ is determined by an L-curve analysis (Hansen, 1992) at each iteration. This recursive filter allows sharper contrasts in the resulting model due to the low dynamic of both model and data in the logarithmic space. From our tests, if the starting model $\sigma_{0}$ is both smooth and close to the true model, then the iterative log inversion converges to a reasonable data misfit after $<10$ iterations.

\section{RESULTS}

\section{D inversion of synthetic VTEM data}

We simulate step-off $B_{z}$ transient responses for the inloop configuration using ArjunAir. We generate three lines of synthetic data as if they were acquired with a VTEM system at the nominal $45 \mathrm{~m}$ clearance. We simulate a transmitter loop of $26 \mathrm{~m}$ diameter and a receiver of $1.1 \mathrm{~m}$ diameter, recording 27 measurements from $t_{1}=83$ $\mu \mathrm{s}$ to $t_{2}=7.8 \mathrm{~ms}$. Synthetic data sets are computed for three models with increasing complexity: the first one is a weakly $2 \mathrm{D}$ conductive medium shown at the top of Figure 4 ; the second one is a clear $2 \mathrm{D}$ conductive body buried inside a uniform medium ground (left column of Figure 5); and the last one is a quasi-horizontal conductive layer embedded beneath an inclined ground (right column of Figure 5). The latter contains topography in addition to conductivity variations.

The first model contains a shallow conductive layer of $500 \mathrm{mS} / \mathrm{m}$ and varying thickness over a resistive half-space of $20 \mathrm{mS} / \mathrm{m}$. The data consist of $36 \mathrm{TEM}$ soundings acquired every $50 \mathrm{~m}$ at a height of $45 \mathrm{~m}$. In Figure 4, the three panels beneath of the true model show the inverted models using respectively, 1D inversion with vertical constraints (VCI), standard 2D Born inversion (AIM) and empirical $2 \mathrm{D}$ inversion. The $1 \mathrm{D}$ inversion fails to reproduce the strong lateral changes occurring at the positions $200 \mathrm{~m}, 900 \mathrm{~m}$ and $1100 \mathrm{~m}$ in the true model. In this case, 1D inversion using lateral constraints (LCI or SCI) (Viezzoli et al., 2008) should provide better results. Using the $2 \mathrm{D}$ interpretations, it is possible to remove the $2 \mathrm{D}$ artefacts without strong regularisation; this is shown in the two bottom graphs of Figure 4.

The second model (Figure 5, top left) is an infinite (strike is the y-direction) prism with a $100 \mathrm{~m} \mathrm{x} 100 \mathrm{~m}$ cross sectional area and a conductivity of $200 \mathrm{mS} / \mathrm{m}$, situated at a depth of $50 \mathrm{~m}$ below the surface. The prism is embedded in a homogeneous half-space with a conductivity $5 \mathrm{mS} / \mathrm{m}$. The reminder of Figure 5 (left column) shows the resulting images from 1D VCI inversion, the 2D Born inversion using an equivalent homogeneous half-space to compute the sensitivity and the 2D empirical inversion. The results show that the 1D inversion or $2 \mathrm{D}$ standard Born inversions fail to reproduce the true model, while the empirical inversion yields a better reconstruction.

$2 \mathrm{D}$ inversion allows (or forces) us to take into account the influence of topography on TEM response. In our processing, we evaluate the angle $\phi$ averaging the local slope over a distance equal to the early-time footprint. Then, the magnetic response is divided by the factor $\cos ^{2} \phi$ to model both the inclination of the receiver and the incidence of the primary magnetic field. In addition, in the inversion, we use a sensitivity function rotated in such a way that the modelled eddy currents are parallel to the ground surface. We tested the three inversion methods on synthetic data computed from the third model that has variation of topography. The model sections obtained for the different methods are presented in the right column of Figure 5. The 2D empirical inversion reproduces well the true model, and avoids the "pant-leg" artefact usually encountered with 1D stitched sections. 

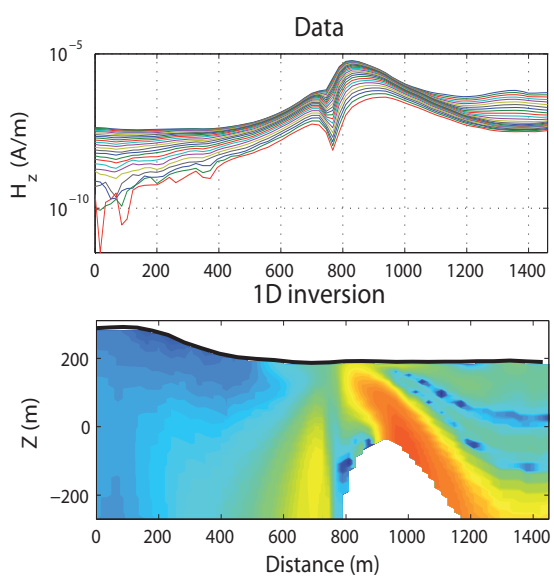

1D approximate data misfit
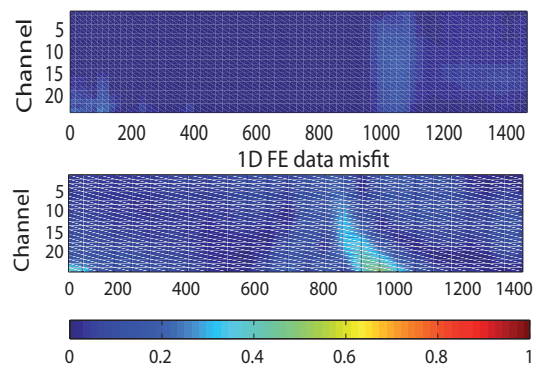
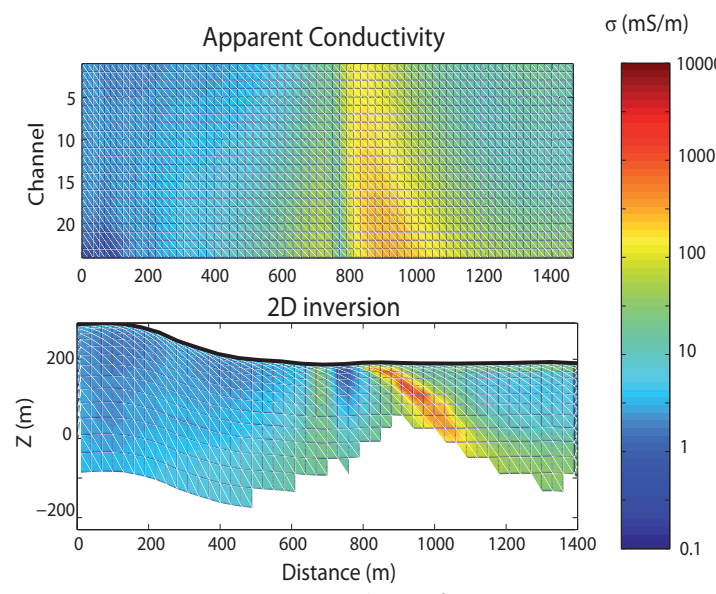

$2 \mathrm{D}$ approximate data misfit
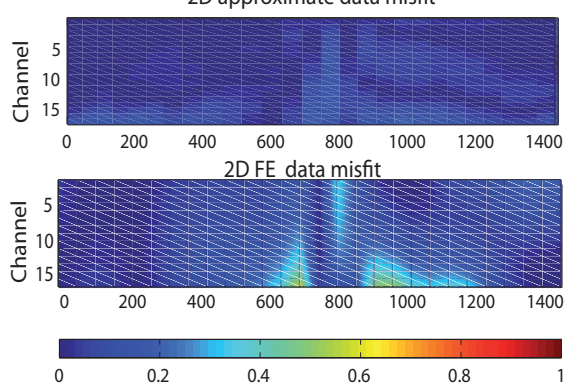

Figure 6: Inversion of VTEM data acquired over the lake Minowean area (Canada). From top to bottom: observed data in term of $H_{z}$ (left) and $\sigma_{a}$ (right), cross section after $1 D$ inversion (left) and $2 D$ inversion (right), and the corresponding approximate data misfit and finite element data misfit.

\section{D inversion of VTEM data}

We apply the 2D empirical inversion method to a helicopterborne TEM data set (VTEM) acquired over the lake Minowean area in Canada. The aim of the survey is to detect and characterize a conductive sheet of graphite beneath the lake. A comparative study of different electrical methods has been presented by Smith et al. (2011) in a similar context. Graphite layers are characterized by strong contrasts of conductivity compared to their host rocks. Moreover, there may be a polarization effects biasing the late time response. We present this challenging case to identify some limitations of the empirical inversion. The transmitter is a four-turn loop, with a diameter of $26 \mathrm{~m}$. The VTEM waveform is a quasi-square function with a maximum peak of $200 \mathrm{~A}$ and a duration of $8.32 \mathrm{~ms}$. The pulse repetition rate is $30 \mathrm{~Hz}$. We invert the vertical component of the magnetic field. Each sounding is composed of 24 channels from $t_{1}=120 \mu \mathrm{s}$ to $t_{24}=6.5 \mathrm{~ms}$. First, the VTEM data (given in $\mathrm{pV} . \mathrm{ms} / \mathrm{A} / \mathrm{m}^{4}$ ) are converted to the equivalent magnetic field (in $\mathrm{A} / \mathrm{m}$ ) which would have been generated by 1 turn-loop and a maximum current peak of $1 \mathrm{~A}$. Then, the apparent conductivity is computed looking in a table of VTEM responses (pure step response corresponding to 1 turn-loop and $1 \mathrm{~A}$ current convolved with the normalized waveform). In Figure 6, we show the results for data acquired at the west border of the lake. The data are displayed as vertical magnetic field profiles (top left) and as an apparent conductivity pseudosection (top right). A visual inspection of the data reveals a large response on both $H_{z}$ and $\sigma_{a}$ that corresponds to a highly conductive body located near $x=800 \mathrm{~m}$. The airborne system, on its flight path, detects the conductive body before passing over it. As a consequence, the 1D inversion images this feature as a deep inclined conductive zone. In the second graph of the left column, the 1D inversion shows the "pant leg" artefact which is often encountered when a 1D inversion is performed in case of high lateral changes of conductivity. The lower limit of imaging corresponds to the apparent depth of investigation as estimated from the late-time channel. The $2 \mathrm{D}$ inversion result is shown in the second graph of the right column. For that case, the lower limit of imaging is derived from the integrated sensitivity matrix (for explanation, see Zhdanov (2009)). Because of polarization and strong scattering effects, the 2D empirical inversion could only fit responses from the first 15 time channels. Indeed, while the $1 \mathrm{D}$ methods are able to fit all the data even in the presence of polarisation or scattering, $2 \mathrm{D}$ inversion cannot do it. The $2 \mathrm{D}$ model contrasts with the stitched 1D results. The conductive body appears as a thinner inclined plate without the $2 \mathrm{D}$ artefacts. In order to estimate the performance of each method, we show the data misfits computed with both the approximate forward modeling and the 2.5D finite element (FE) forward modeling. The 1D and 2D inversions show similar errors. 
However, comparing to geological information in the area, the small thickness of the graphite sheet in the 2D image is more realistic than the larger one obtained in the $1 \mathrm{D}$ inversion.

\section{CONCLUSION}

The sensitivity of geophysical EM measurements depends on both the subsurface current distribution induced by the source and the relative position of the receiver. For in-loop configurations, we compared realistic 2D sensitivities computed using numerical forward modeling to a $2 \mathrm{D}$ pseudo-sensitivity derived from the Born approximation for homogeneous media. Our results show that standard approximate mapping methods do not describe well the pattern of eddy currents, even for low conductivity perturbation. We show that using a Born approximation based on equivalent homogeneous theory is not effective for $2 \mathrm{D}$ inversion. A $2 \mathrm{D}$ contrast of conductivity is equivalent to an infinite prism perpendicular to the profile which perturbs the EM response much more than a 3D body. We suggest that the AIM method would work better for 3D inversion in the case of a small 3D body with low conductivity contrast.

For fast 2D inversion, we suggest the use of the empirical sensitivity function presented in this paper. The resulting method is very fast, and it is almost as practical as 1D inversion for processing large amounts of data. For long profile of data, we suggest using this method with a sliding inversion window of around $2-3 \mathrm{~km}$ width (the computation time is $1 \mathrm{~min} / \mathrm{km}$ with one processor of 2.39 $\mathrm{GHz}$ and 3.45 Go of RAM).

Our mathematical model describes to first order the physics of the in-loop TEM measurement. Application to real and synthetic data show that it describes the behaviour of eddy currents better than 1D approximations and improves the quality of ground imaging. Nevertheless, the empirical approach fails to reproduce the real inclination of thin conductive sheets. Indeed, this property is controlled by the factors 4 and 6 in the exponential function (equation 17) which may be dependent on the conductivity of the host medium. In future, it is important to better define the empirical mathematical model in order to improve imaging.

We suggest that the new 2D method should replace $1 \mathrm{D}$ inversion for fast processing of airborne data. It also provides a better starting model for more rigorous inversion schemes. Using numerical modeling, it is possible to estimate the $2 \mathrm{D}$ sensitivity for all the configurations and to approximate it by simple mathematical functions. This suggests using the same approach as shown here to estimate the approximate 2D sensitivity of other EM systems. At this time, our method is limited to the in-loop, vertical magnetic field geometry, notably the AEM geometry with the smallest lateral sensitivity. Adopting our method to dual loop and/or horizontal receiver components systems might improve the final 2D model.

\section{ACKNOWLEDGMENTS}

This paper results from a Joint Research Project between AREVA-NC and CNRS UMR 7516. The authors thank AREVA Mining Business Group for financial support to J.G. as PhD student and for providing VTEM data. We thank the two anonymous reviewers, as well as N.B. Christensen and A.A. Pfaffhuber for their instructive comments which considerably improved the quality of the manuscript. We also had numerous discussions with our colleague Pierre Keating.

\section{APPENDIX A ELECTRICAL FIELD WITHIN A HOMOGENEOUS HALF-SPACE}

The EM Field caused by a horizontal loop source over a horizontal half-space is derived for the air layer in Ward and Hohmann (1987) using the Schelkunoff potential vector. In this appendix, we show how to determine the electrical field within the half-space by using the same approach.

In the air $(n=0)$, the Schelkunoff potential vector $\tilde{F}_{1}$ follows the wave equation which is given in the Frequency domain by:

$\tilde{F}_{0}\left(\omega, k_{x}, k_{y}, z\right)=\left(A_{i}+A_{r}\right) \frac{e^{-u_{0} z}}{2 u_{0}}=i \omega \mu_{0} M e^{-u_{0} h}\left(1+r_{T E}\right) \frac{e^{-u_{0} z}}{2 u_{0}}$,

where $u_{0}$ is the wave number in the air $\left(u_{0}^{2}=k_{x}^{2}+k_{y}^{2}-\right.$ $\left.\omega^{2} \mu_{0} \epsilon_{0}\right), M$ is the magnetic moment of the source and $r_{T E}$ is the reflection coefficient of the wave at the interface between the air and the ground. Within the half-space $(n=1)$, the transmitted wave is given by:

$$
\tilde{F}_{1}\left(\omega, k_{x}, k_{y}, z\right)=A_{t} \frac{e^{-u_{1} z}}{2 u_{1}},
$$

where where $u_{1}$ is the wave number in the ground $\left(u_{1}^{2}=\right.$ $\left.k_{x}^{2}+k_{y}^{2}+i \omega \mu_{0} \sigma_{1}\right)$ and $A_{t}$ is the amplitude factor depending of both the incident wave amplitude and the transmission coefficient:

$$
A_{t}=t_{T E} A_{i} .
$$

By applying the boundary conditions at $z=0$, one obtains the following problem:

$$
\left\{\begin{array}{l}
\frac{A_{i}}{u_{0}}\left(1+r_{T E}\right)=\frac{A_{t}}{u_{1}} \\
A_{i}\left(1-r_{T E}\right)=A_{t}
\end{array},\right.
$$

from what one can derive $A_{t}$ :

$$
A_{t}=A_{i} \frac{2 u_{1}}{u_{0}+u_{1}}
$$

By analogy with equation (A-3), one can obtain the expression for transmission coefficient:

$$
t_{T E}=\frac{2 u_{1}}{u_{0}+u_{1}} .
$$

Thus, the Schelkunoff potential $\tilde{F}_{1}$ within the half-space is given by the following equation:

$$
\tilde{F}_{1}\left(\omega, k_{x}, k_{y}, z\right)=i \omega \mu_{0} M e^{-u_{0} h} t_{T E} \frac{e^{-u_{1} z}}{2 u_{1}} .
$$


For the case of a circular loop source with the radius $a$, one can use the spatial inverse Fourier transform in cylindrical coordinates; the equation (A-7) becomes:

$$
F_{1}(\omega, \rho, z)=\frac{i \omega \mu_{0} I a}{2} \int_{0}^{\infty} \frac{t_{T E}}{u_{1}} e^{-u_{0} h} e^{-u_{1} z} J_{1}(\lambda a) J_{0}(\lambda \rho) d \lambda .
$$

The horizontal electrical field $E_{\phi}$ for the TE mode is derived from the expression of the potential vector $F_{1}$ by using the following equation:

$$
\begin{aligned}
& E_{\rho}=-\frac{1}{\rho} \frac{\partial F_{1}}{\partial \phi} \\
& E_{\phi}=\frac{\partial F_{1}}{\partial \rho} \\
& E_{z}=0
\end{aligned} .
$$

For a homogeneous and isotropic medium, the electrical field is invariant with regards to $u_{\phi}$. Therefore, following (A-9), the radial component of the electrical field $E_{\rho}$ is null. The electrical field is oriented along $u_{\phi}$ only. By using (A-9), one can write the following equation for the impulse response of the electrical field:

$$
E_{\phi}(\omega, \rho, z)=\frac{-i \omega \mu_{0} I a}{2} \int_{0}^{\infty} \frac{t_{T E}}{u_{1}} e^{-u_{0} h} e^{-u_{1} z} \lambda J_{1}(\lambda a) J_{1}(\lambda \rho) d \lambda .
$$

The step response for the electrical field is obtained by dividing the last expression by $-i \omega$.

\section{REFERENCES}

Aster, R., B. Borchers, and C. Thurber, 2005, Parameter Estimation and Inverse Problems: Elsevier Academic Press.

Auken, E., S. Violette, N. D'Ozouville, B. Desfontaines, K. Sorensen, A. Viezzoli, and G. de Marsily, 2009, An integrated study of hydrogeology of volcanic islands using helicopter borne transient electromagnetic: Application in the Galapagos Archipelago: C.R. Geosciences, 341, 899-907.

Baldridge, W., G. Cole, B. Robinson, and G. Jiracek, 2007, Application of Time-Domain airborne electromagnetic induction to hydrologic investigations on the Pajarito Plateau, New Mexico, USA: Geophysics, 72, B31-B45.

Chen, J., and A. Raiche, 1998, Inverting AEM data using a damped eigenparameter method: Exploration Geophysics, 29, 128-132.

Christensen, N., 1995, 1D Imaging of central loop transient electromagnetic soundings: Journal of envir. engin. geophysics, 0, 53-66.

, 2002, A generic 1-D imaging method for transient electromagnetic data: Geophysics, 67, 438-447.

Cox, L., G. Wilson, and M. Zhdanov, 2010, 3D inversion of airborne electromagnetic data using a moving footprint: Exploration Geophysics, 41, 250-259.

Danielsen, J., E. Auken, F. Jorgensen, V. Sondergaard, and K. Sorensen, 2003, The application of transient electromagnetic method in hydrogeophysical surveys: Journal of Applied Geophysics, 53, 181-198.

Eaton, P., and G. Hohmann, 1987, A rapid inversion technique for transient electromagnetic soundings: Physics of the Earth and Planetary Interiors, 53, 384-404.

Farquharson, C., D. Oldenburg, and Y. Li, 1999, An approximate inversion algorithm for time-domain electromagnetic survey: Journal of Applied Geophysics, 42, 71-80.

Gòmez-Treviño, E., 1987a, A simple sensitivity analysis of time-domain and frequency-domain electromagnetic measurements: Geophysics, 52, 1418-1423.

$\longrightarrow, 1987 \mathrm{~b}$, Nonlinear integral equations for electromagnetic inverse problems: Geophysics, 52.

Guillemoteau, J., P. Sailhac, and M. Behaegel, 2011, Regularisation strategy for the layered inversion of Airborne TEM data: application on data aquired over the basin of Franceville (Gabon): Geophysical Prospecting, 59, 1132-1143.

Hansen, P., 1992, Analysis of Discrete Ill-Posed Problems by Means of the L-Curve: SIAM Review, 34, 561-580.

— , 2010, Discrete Inverse Problem: Insight and Algorithms: SIAM, Philadelphia.

Huang, H., and G. Palacky, 1991, Damped least-square inversion of time-domain airborne EM data based on singular value decomposition: Geophysical Prospecting, 39, 827-844.

Huang, H., and J. Rudd, 2008, Conductivity-depth imaging of helicopter-borne TEM data on a pseudolayer half- 
space model: Geophysics, 73, F115-F120.

Keating, P., and D. Crossley, 1990, The inversion of timedomain airborne electromagnetic data using the plate model: Geophysics, 55, 705.

Kirkegaard, C., T. Sonnenborg, E. Auken, and F. Jørgensen, 2011, Salinity distribution in heterogeneous coastal aquifers mapped by airborne electromagnetics: Vadose Zone Journal, 10, 125-135.

Ley-Cooper, A., J. Macnae, and A. Viezzoli, 2010, Breaks in lithology: Interpretation problems when handling 2D structures with a 1D approximation: Geophysics, 75, 179-188.

Macnae, J., A. King, N. Stolz, A. Osmakoff, and A. Blaha, 1998, Fast AEM data processing and inversion: Exploration Geophysics, 29, 163-169.

Nabighian, M. N., 1979, Quasi-static transient response of a conducting half-space- An approximate representation: Geophysics, 44, 1700-1705.

Nabighian, M. N., and J. Macnae, 1988, Time domain electomagnetic prospecting methods: in Nabighian, M.N., Ed.,Investigation in geophysics no. 3, Soc. Expl. Geophys.

Oldenburg, D., and R. Ellis, 1991, Inversion of geophysical data using an approximate inverse mapping: Geophys. J. Int., 105, 325-353.

Palacky, G., 1981, The airborne electromagnetic method as a tool of geological mapping: Geophysical Prospecting, 29, 60-88.

Pfaffhuber, A., S. Monstad, and J. Rudd, 2009, Airborne electromagnetic hydocarbon mapping in Mozambic: Exploration Geophysics, 40, 1-9.

Raiche, A., 2008, The P223 software suite for planning and interpeting EM surveys: Previews, 132, 25-30.

Reid, J., and J. Macnae, 1998, Comments on the electromagnetic smoke ring concept: Geophysics, 63, 19081913.

Sattel, D., 2005, Inverting airborne electromagnetic (AEM) data with Zohdy's method: Geophysics, 70, G77-G85.

Siemon, B., A. Christiansen, and E. Auken, 2009, A review of helicopter-borne electromagnetic methods for ground water exploration: Near Surface Geophysics, 7, 629-646.

Smith, R., R. Koch, G. Hodges, and J. Lerieux, 2011, A comparison of airborne electromagnetic data with ground resistivity data over the Midwest deposit in the Athasbasca basin: Near Surface Geophysics, 9, 319330 .

Smith, R., G. Wood, and B. Powell, 2010, Detection of alteration at the Millenium uranium deposit in the Athabasca Basin: A comparison of data from two airborne electromagnetic systems with ground resistivity data: Geophysical Prospecting, 58, 1147-1158.

Tartaras, E., M. Zhdanov, K. Wada, A. Saito, and T. Hara, 2000, Fast Imaging of TDEM data based on Sinversion: Journal of Applied Geophysics, 43, 15-32.

Vallee, M. A., and R. Smith, 2009, Inversion of airborne time-domain electromagnetic data to a 1D structure using lateral constraints: Near Surface Geophysics, 7, 6371.

Viezzoli, A., A. Christiansen, E. Auken, and K. Sorensen, 2008, Quasi-3D modeling of airborne TEM data by spatially constrained inversion: Geophysics, 73, F105F113.

Ward, S., and G. Hohmann, 1987, Electromagnetic theory for geophysical applications: in Nabighian, M.N., Ed., Investigation in geophysics no. 3, Soc. Expl. Geophys.

Wilson, G., A. Raich, and F. Sugeng, 2006, 2.5D inversion of airborne electromagnetic data: Exploration Geophysics, 37, 363-371.

Wolfgram, P., and H. Golden, 2001, Airborne EM applied to sulphide nickel- Examples and analysis: Exploration Geophysics, 32, 136-140.

Wolfgram, P., and G. Karlik, 1995, Conductivity-depth transform of GEOTEM data: Exploration Geophysics, 26, 179-185.

Wolfgram, P., D. Sattel, and N. Christensen, 2003, Approximate $2 \mathrm{D}$ inversion of AEM data: Exploration Geophysics, 34, 29-33.

Zhdanov, M., 2009, Geophysical electromagnetic theory and methods: Elsevier, Amsterdam.

Zhdanov, M., D. Pavlov, and R. Ellis, 2002, Localized S-inversion of time-domain electromagnetic data: Geophysics, 67, 1115-1125.

Zohdy, A., 1989, A new method for the automatic interpretation of Schlumberger and Wenner sounding curves: Geophysics, 54, 245-253. 